Lung adenocarcinoma (LA) is the most common cause of cancer-related death worldwide. Despite the advances over last decade in new targeted therapies, cancer genetics, diagnostics, staging, and surgical techniques as well as new chemotherapy and radiotherapy protocols, the death rate from LA remains high. The tumour microenvironment is composed of several cytokines, one of which is transforming growth factor $\beta 1$ (TGF- $\beta 1$ ), which modulates and mediates the expression of epithelial-mesenchymal transition (EMT), correlated with invasive growth in LAs, and exhibits its pleiotropic effects through binding to transmembrane receptors T $\beta \mathrm{R}-1$ (also termed activin receptor-like kinases ALKs) and T $\beta R-2$. Accordingly, there is an urgent need to elucidate the molecular mechanisms associated with the tumoural spreading process and therapeutic resistance of this serious pathology. In this review, we briefly discuss the current role of contextual signal TGF- $\beta 1$ inducer of epithelial mesenchymal transition in metastatic lung adenocarcinoma patients with brain metastases, and give an overview of our current mechanistic understanding of the TGF- $\beta 1$-related pathways in brain metastases progression, TGF- $\beta 1$ pathway inhibitors that could be used for clinical treatment, and examination of models used to study these processes. Finally, we summarise the current progress in the therapeutic approaches targeting TGF- $\beta 1$.

Key words: TGF- $\beta 1$, EMT, lung adenocarcinoma, brain metastasis, pathology.

Contemp Oncol (Pozn) 2019; 23 (4): 187-194 DOl: https://doi.org/10.5114/wo.2019.91543

\section{The role of contextual signal TGF- $\beta 1$ inducer of epithelial mesenchymal transition in metastatic lung adenocarcinoma patients with brain metastases: an update on its pathological significance and therapeutic potential}

Kelvin Piña Batista ${ }^{1}$, Kenia Álvarez Reyes De Piña ${ }^{2}$, Aida Antuña Ramos ${ }^{1}$,
Ivan Fernandez Vega ${ }^{1}$, Antonio Saiz ${ }^{1}$, Marco A. Álvarez Vega ${ }^{1}$

'Department of Neurosurgery, Central University Hospital of Asturias, Oviedo, Spain ${ }^{2}$ Hospital Vital Álvarez Buylla, Mieres, Spain

\section{Introduction}

Lung adenocarcinoma (LA) is the most common cause of cancer-related death worldwide [1-8]. Despite the advances over the last decade in new targeted therapies, cancer genetics, diagnostics, staging, and surgical techniques as well as new chemotherapy and radiotherapy protocols, the death rate from LA remains high [3, 4, 9-11]. A number of strategies have been employed. Early LA is asymptomatic, and the vast majority of patients are diagnosed at an advanced stage. LA is an umbrella term, under which fall multiple histologic variants that are mainly composed of an array of heterogeneous molecular subtypes, most of them characterised by a single oncogenic alteration $[1,3,8]$. From a clinical and pathological standpoint, the vast majority of LAs are diagnosed using a single adenocarcinoma marker (e.g. TTF-1 or mucin) and are grouped histologically according to tissue morphology as epithelial lung tumours, which are further subclassified into: lipidic adenocarcinoma; acinar adenocarcinoma; papillary adenocarcinoma; micropapillary adenocarcinoma; solid adenocarcinoma; invasive mucinous adenocarcinoma, with its variants mixed invasive mucinous and non-mucinous adenocarcinoma; colloid adenocarcinoma; foetal adenocarcinoma; enteric adenocarcinoma; minimally invasive adenocarcinoma, with two types: mucinous and non-mucinous; and preinvasive lesions: atypical adenomatous hyperplasia and adenocarcinoma in situ, which can be mucinous or non-mucinous [4, 12-14].

LAs arise mostly from distal airways, commonly with glandular histology displaying biomarkers that are consistent with an origin in the distal lung, including thyroid transcription factor 1 (NKX2-1) and keratin [3, 13, 15-17].

Unfortunately, the clinical, radiological, molecular, and pathological spectrum is widely divergent in terms of overall survival and prognosis due to histological heterogeneity of lung adenocarcinomas [4]. Accordingly, there is a critical need to elucidate the molecular mechanisms associated with the tumoural spreading process and therapeutic resistance of this serious pathology.

The tumour microenvironment is composed of several cytokines, one of which is transforming growth factor $\beta 1$ (TGF- $\beta 1$ ), which modulates and mediates the expression of epithelial-mesenchymal transition (EMT), correlated with invasive growth in LAs, and exhibits its pleiotropic effects through 
binding to transmembrane receptors T $\beta R$-1 (also termed activin receptor-like kinases [ALKs]) and T $\beta R$-2. TGF- $\beta$ is expressed on several malignancies, including LAs [9, 18-25].

TGF- $\beta$ signalling promotes the migration of tumour cells to metastasis sites. Humans express three highly potent homologous cytokine isoforms of TGF- $\beta$ consisting of TGF- $\beta$ 1, 2, and 3. In LA carcinogenesis TGF- $\beta 1$ plays an important role in regulating cellular functions such as cell proliferation, differentiation, tumour suppression, epithelial inflammation, cell motility, apoptosis, adhesion, invasion, and immune response [26-29]. Functionally, TGF- $\beta 1$ cytokines exert pro-inflammatory effects in epithelial tumourigenesis. Initially, TGF- $\beta 1$ acts as a tumour suppressor. At later stages, as the tumour grows, TGF- $\beta 1$ is produced by both tumour and stromal cells as a natural response to hypoxic and inflammation [30].

In this review, the current role of contextual signal TGF- $\beta 1$ inducer of epithelial mesenchymal transition in patients with lung adenocarcinoma, and an overview of our current mechanistic understanding of the TGF- $\beta 1$-related pathways in brain metastasis progression-inhibitors that could be used for clinical treatment, as well as an examination of the models used to study these processes, will be briefly discussed. Finally, current progress in the therapeutic approaches targeting TGF- $\beta 1$ will be summarize.

\section{TGF- $\beta 1$ signalling pathway plays a critical role in EMT expression in lung adenocarcinomas}

Several studies have reported that TGF- $\beta 1$ is involved in the tumorigenesis of lung adenocarcinoma cells. The TGF- $\beta$ superfamily, first coined as sarcoma growth factor by de Larco and Todaro in 1978 [5, 31], seems to regulate the transdifferentiation process of EMT. Importantly, TGF- $\beta 1$ protein has been shown to play a critical role in the development of EMT [1, 15, 18, 22, 31-62]. However, despite some excellent published work, the role of TGF- $\beta 1$ on cellular proliferation, invasiveness, immune response, angiogenesis, differentiation, oxidative stress, and metastasis pathophysiology remains to be fully elucidated. EMT is a well-studied biologic phenotypic switching process by which epithelial cells lose their characteristic signals and cell adhesion properties converting into motile mesenchymal cells [9, 19, 22, 47]. Three classes of EMT events have been identified. Type 3 EMTs occur in tumour cells [9, 20].

On the other hand, EMT play a pivotal role in the context; it was shown that TGF- $\beta 1$ coordinates and EMT plays an important role in control of growth, survival, and metastasis $[39,59]$. TGF/EMT signalling, which is mainly mediated by RAS-MAPK, a driver and critical downstream pathway of human carcinomas, and PI3K-AKT/HIF-1a pathways affect gene expression and cell cycle progression through the binding of transcription factors $[19,45]$ such as the E26 transformation-specific (ETS) family, regulating EMT by enhancing the expression of EMT-transcription factors including ZEB1, Snail, VEGF, etc. [19, 42, 63-65]. Cytoplasmic signalling cascades mediated by PI3K-AKT and the GTPase RAC1 or cell division control protein 42 (CDC42) is found to be altered in several stages of tumour progression. MMP-3 increases the level of RAC1b, which gives rise to cellular
ROS, leading to EMT and oxidative damage to DNA, modulating cell survival, and eliciting actin and microtubule cytoskeletal changes, regulation of gene expression, cell polarity, and cell migration $[10,17]$.

The TGF- $\beta 1$ signalling is regarded as an initial, complex, and yet crucial cytokine to induce EMT during cancer progression and metastasis in a Smad2/Smad3 complex-dependent C-terminal phosphorylated downstream manner via ALK-5 receptors, and also dependent on the protein-serine/threonine kinases that participate in the Ras-Raf-MEK5-ERK5 signal transduction cascade and nuclear translocation $[1,15,18,22,25,29,31,33,45,47-49$, 57, 65-72], with positive enhancement of T-cell and cytokine production [73]. Subsequently this Smad2/Smad3 bond forms a special hetero-oligomeric link with common mediator Smad4 giving way to a SMAD2/3/4 translocating complex into the nucleus, where it binds to transcription factors Snail/Slug, Twist, and Zeb, subsequently modulating the transcription of target genes that regulate cellcell and ECM interactions, thus providing a favourable microenvironment for tumour cell spread [18, 47-49, 71, 72, 74]. TGF- $\beta 1$ acts as a pleiotropic factor and cytokine, promoting cell proliferation, survival, motility, scattering, differentiation, and morphogenesis. Physiologically, EMT is responsible for the cell-scattering phenotype. This process involves the disruption of cadherin-based cell-cell contacts and subsequent migration of the dissolved cells into the circulation [75]. High TGF- $\beta$ signalling promoting EMT invasiveness and metastatic growth is achieved by ligand binding to TGF- $\beta$ receptor $I$, which associates with the type I receptor activin receptor-like kinase-5 (ALK-5) through the subsequent phosphorylation of Smad2 and Smad3, which is constitutively regulated by the cytoplasmic protein profilin-2 (PFN2) $[23,75]$. However, a more recent in vivo liver and lung metastasis mouse model study investigating whether PFN2 served specific roles in the progression of human colorectal cancer demonstrated, by reverse transcription-quantitative polymerase chain reaction and western blotting techniques, that PFN2 expression was reduced in metastatic CRC [69].

Molecularly, during embryogenesis TGF- $\beta$ cytokine leads several biological processes such as cell cycle and apoptosis, EMT, and extracellular matrix (ECM) regulation. Recent studies investigating molecular mechanisms and epigenetic alterations of carcinogenesis have indicated that Smad6 antagonises Smad2-4 complex activation by competing with Smad2-4 binding to the activated TGF- $\beta 1$ receptor [76] and that bone morphogenetic protein (BMP)-mediated SMAD signalling requires an arginine methylation step for the removal of an otherwise signalling-inhibiting SMAD6 protein ahead of transphosphorylation of the TGF- $\beta /$ BMP receptor kinases $[3,13,76]$. Furthermore, emerging evidence supports the theory that SMAD6 inhibits BMP signalling by interacting with homeobox C8 in the nucleus, and Smad6 antagonises TGF- $\beta 1$ in the cytoplasm through the formation of a stable complex with TGF- $\beta$ R1. In contrast, SMAD7 (mothers against decapentaplegic homolog 7) inhibits TGF- $\beta$, activin, and BMP signalling. Smad7 interacts with TGF- $\beta$ R1 to inhibit R-smad activation and is commonly found in patients with chronic 


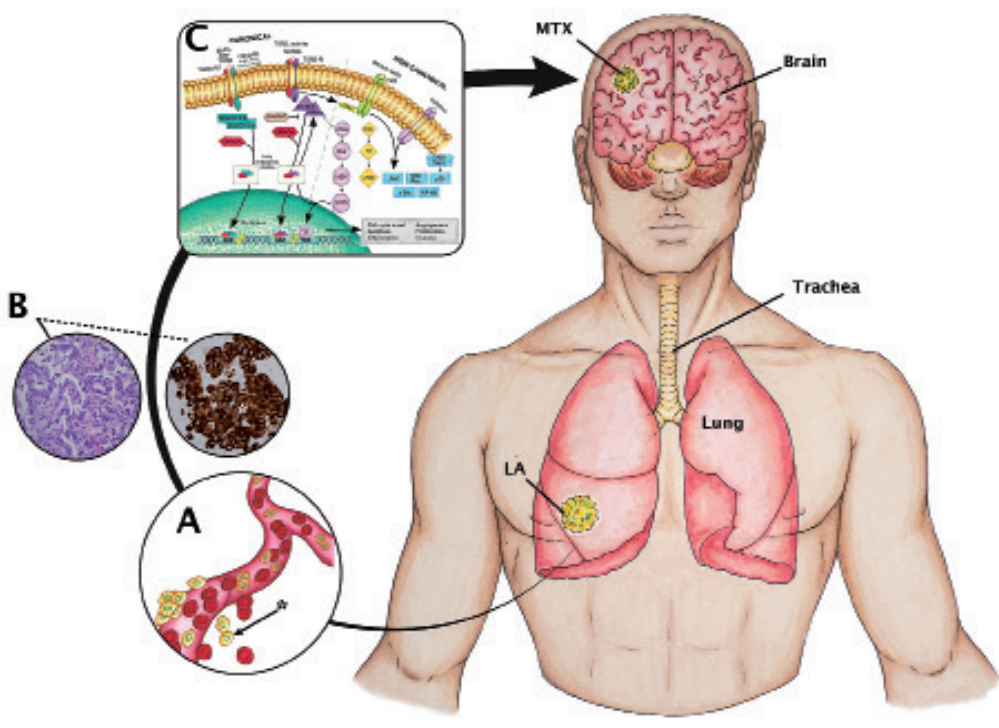

Fig. 1. Canonical (Smad-dependent) and non-canonical (Smad-independent) pathways. An oversimplified and modified (from [30]) scheme depicting the TGF- $\beta$ pathway as well as a model of cerebral metastasis from lung adenocarcinoma. A) Disaggregation of cells from primary tumour mass to a particular metastatic niche is mediated mainly through integrins and proteases expressed at the surface of cancer cells, vasculature, and stromal cells [48]. In summary, cells [*] break through the vascular basement membrane via the expression of cellular matrix metalloproteinase activity - MMP2 and MMP9. Many angiogenic factors secreted by both tumour and stromal cells, such as VEGF and platelet-derived growth factor $\beta$ (PDGF- $\beta$ ), which are transcriptionally directly driven by hypoxia-inducible factor 1 or 2 (HIF1-HIF2). B) Lung adenocarcinoma is commonly defined as a slow-growing cancer that arises mostly from distal airways, typically with glandular histology displaying biomarkers that are consistent with an origin in the distal lung, including thyroid transcription factor 1 (NKX2-1) and keratin (7) with complex early diagnosis because it usually involves the periphery of the lung and has scarce symptoms, promoting early metastasis. C) The TGF- $\beta 1$ signalling is regarded as an initial, complex, and yet crucial cytokine to induce EMT during cancer progression and metastasis (20) in a Smad2/Smad3 complex-dependent C-terminal phosphorylated downstream manner via ALK-5 receptors, and also dependent on the protein-serine/threonine kinases that participate in the Ras-Raf-MEK5-ERK5 signal transduction cascade and nuclear translocation

inflammatory conditions of the colon and also enhances the invasiveness of non-small-cell lung cancer cells. Recent studies have shown that Smad7 can be promoted and targeted by micro-RNAs (miR), such as miR-106b-25 and miR-21-5p cluster, during EMT in breast cancer development and NSCLC proliferation, respectively [77, 78].

Although the importance of epigenetic mechanisms regulating TGF- $\beta 1$ has been recognised, several groups have reported genome-wide analyses of the binding patterns of TGF- $\beta 1$ receptor-regulated Smads in various cancer cell lines and embryonic stem cell-derived cells. Various Smad-binding profiles have been revealed in different cell types, indicating that the cell environment is of paramount importance for the response to signalling [3-5, 11, 23, 36, 43, 44, 48, 67, 77, 79-84].

The combination of in vitro cell-based or in vivo mousebased models has been widely used to interrogate the dynamic behaviour of TGF- $\beta 1$ signalling networks. However, the majority of models have been created by directly inactivating the expression of negative feedback pathway regulators, such as the E3 ubiquitin ligase SMURF2 and SMAD7 of the TGF- $\beta$ superfamily pathway $[17,32-36,77$, 78, 85-87]. Considering its inhibitory roles in the TGF- $\beta 1$ receptor, inhibitory SMAD proteins may play a critical role by inhibiting the development of endothelial cells. Furthermore, distinctive canonical (Smad-dependent) and non-canonical (Smad-independent) (Fig. 1) TGF- $\beta$ super- family member inhibitors have been identified using human and animal models [60] such as GDF15 (identified as a critical downstream mediator of CDP138, and described as a CDK5 binding partner that regulates cell proliferation and migration), which attenuates the TGF- $\beta / \mathrm{Smad}$ signalling pathway, leading to impaired radio-resistance and metastasis in lung cancer [10]; CK2 (formally named casein kinase 2) inhibitor CX-4945 affects the TGF- $\beta 1$-induced cadherin switch and the activation of the smad, non-smad, and focal adhesion signalling pathways, avoiding the migration and invasion of A549 human lung epithelial adenocarcinoma cells [61]; Bcl-xL, an anti-apoptotic and probably antioxidant protein overexpressed in LAs, has been demonstrated to neutralise the pro-apoptotic functions of $\mathrm{Bcl}$-2-associated X protein (Bax) and Bcl-2-associated death promoter (Bad) through mitochondrial membrane permeability integrity, which mechanistically preclude pro-caspace- 9 activation and cytochrome $c$ release [50, 88, 89], among other factors. However, although these inhibitor pathways are attractive therapeutic targets in LAs [90], mounting evidence indicates that Smad/ TGF- $\beta$ /EMT axis suppression, like most complex biological pathways, leads to compensatory over-activation of upstream pathways, resulting in sustained inhibition of the anti-angiogenic, anti-proliferative, and anti-metastatic effects of the recognised inhibitors. In order to examine and to understand the mechanism of the therapeutic po- 
tential of targeting TGF- $\beta$, it is necessary to elucidate the full spectrum of feedback loops that are unleashed by suppression of the smad/ TGF- $\beta /$ EMT axis $[60,81,91]$.

\section{Carcinogenesis and evolution. Brain metastases from lung adenocarcinoma}

Metastatic tumours are the most common neoplasms encountered in the brain. Brain metastases most commonly arise from lung, breast, genitourinary tract, and melanoma [41, 42, 55, 92]. Once brain metastases have developed, survival is commonly short, sometimes secondary by a scarce follow-up.

Brain metastasis is a multistep process that involves multiple interactions between the tumour cells and the matrix proteins, with the microenvironment playing a key factor, characterised by abnormal changes at the cellular, genetic, and epigenetic levels. Initiation and development of lung adenocarcinoma is partially attributed to the dysregulation and aberrant expression of chromosomes and proto-oncogenes, ranging from simple structural rearrangements and gene amplifications to losses or gains of entire chromosomes, which lead to cell growth, metastasis, and other tumour progressions. Several protooncogenes have been reported in LAs, such as ALK, protooncogene tyrosine-protein kinase (ROS1), c-Met, and RET proto-oncogene $[58,67,70,93]$. Furthermore, it has been established that many miRNAs are down-regulated or up-regulated in certain types of cancers. MicroRNAs (miRNAs) are short endogenous non-coding RNAs implicated in the regulation of gene expression of many relevant physiological processes, including cell proliferation, cell death, and stress responses [35], and thus key drivers of pathological control. It is estimated that up to $30-50 \%$ of all human protein-coding genes are regulated by miRNAs [94].

Mounting evidence suggests that miRNAs are deregulated in various human cancers, including LAs, by regulating expression of multiple target genes [52]. They function as oncogenes or tumour suppressor genes. In the context of cancer, a recent study using TGF- $\beta 1$ in different concentrations to induce EMT in lung cancer A549 cells demonstrated that the lung cancer A549 cells became elongated and the cell-cell junction became loose after EMT $[24,29]$, resulting in E-cadherin downregulation and the mesenchymal markers vimentin and fibronectin being up-regulated, supporting the theory that EMT has an effect on the expression of miRNAs, and regulating metastasis of lung cancer cells via EMT [34]. To investigate the function of miR-26a in the development of lung cancer, based on recent published data, in order to find prognosis-related miRNA, miR-29c isoform, a member of miR-29 family, aberrantly expressed in different types of human cancers and functionally involved in cell proliferation, cell cycle, apoptosis, and metastasis, has been demonstrated to revert aberrant methylation by targeting DNA methyltransferases $3 \mathrm{~A}$ and $3 \mathrm{~B}$ and inhibits cell proliferation, migration, and invasion in cell lines by targeting integrin $\beta 1$ and matrix metalloproteinase 2 expression [74, 95]. Interestingly enough, miR-29c may target different genes and pathways to inhibit tumour growth and metastasis, implicating miR-29c as a novel promising prognostic biomarker as well as a therapeutic target to provide clinical benefit, through modulating proliferation and migration/invasion of LA cells by VEGFR-2l [29].

Notably, increasing evidence has demonstrated that TGF- $\beta$ through EMT induces the expression of matrix metalloproteinases on both endothelial cells and tumour cells, which then facilitates the differentiation into stromal stem cells such as pericytes, and is able to create a tissue environment permissive to the metastatic dissemination and colonisation of distant organs [36, 40]. More specifically, cells break through the vascular basement membrane via the expression of cellular matrix metalloproteinase activity - MMP2 and MMP9. Many angiogenic factors secreted by both tumour and stromal cells in the brain, such as VEGF and platelet-derived growth factor $\beta$ (PDGF- $\beta$ ) are transcriptionally directly driven by hypoxia-inducible factor 1 or 2 (HIF1-HIF2) [19, 29, 73].

Additionally, acidosis, cellular stress, and hypoxia lead to decreased expression of E-cadherin and occludins, resulting in less cell adhesion and entry of the dissolved or circulating tumour cells (CTCS) intro the bloodstream, some of which express the EMT phenotype [20]. E-cadherin is a calcium-dependent cell adhesion glycoprotein abundantly expressed by surface epithelial cells that function as an inhibitory factor for malignant transformation, cell dissociation, and metastasis. Therefore, E-cadherin is a rate-limiting step of invasion and differentiation of cells, downregulated in epithelial cancers, categorised as a marker for EMT. In fact, EMT is well known as a cellular program characterised by loss of an epithelial gene expression signature, such as E-cadherin, and gain of genes that define mesenchymal features, such as vimentin and neural cadherin (N-cadherin) $[9,18,34,63,96,97]$.

At epigenetic level, increased Smad2 and Smad3 expression operates in the lung adenocarcinomas and brain metastasis, enhancing TGF- $\beta 1$ and the production of angiogenic factors such as VEGF [23, 83, 95]. Some investigators have also focused on the role of vascular basement membrane (VBM) in the pathogenesis of brain metastasis [92, 98]. Concordantly, VBM promoted adhesion and invasion of malignant cells. Disaggregation of cells from the primary tumour mass to a particular metastatic niche is mediated mainly through integrins and proteases expressed at the surface of cancer cells, vasculature, stromal cells, etc. [92]. The $\beta 1$ subunit of integrin is an adhesion molecule involved in cell survival and cancer resistance to radio and chemotherapy. Cooperation between $\beta 1$ integrin and c-Met regulates tyrosine kinase inhibitor resistance in lung cancer. Blockade of the $\beta 1$ integrin subunit in tumour cells prevented adhesion to VBM and attenuated metastasis establishment and growth in vivo $[29,34,38,41,58]$.

Recent insights into the brain tumour microenvironment have begun to uncover the close association between metastatic cells and the blood-brain barrier, by disrupting the endothelium through the vascular basement membrane to gain entry into the circulation and 
promoting tumour cell dedifferentiation transcriptionally. The VBM serves also as a reservoir for growth factors, such as TGF- $\beta 1$ and vascular endothelial growth factor (VEGF), which reduce the endothelial barrier function by disrupting the E-cadherin- $\beta$-catenin complex and therefore favouring endothelial cell junction opening [26, 84, 99].

Interestingly, bevacizumab is a humanised MAb targeting VEGF. The inhibition of VEGF signalling via bevacizumab treatment may normalise tumour vasculature, promoting a more effective delivery of chemotherapy agents. A randomised phase III trial (ECOG 4599) combining paclitaxel and carboplatin with or without bevacizumab in patients with advanced LA found a significant improvement in median survival for patients in the bevacizumab group, with a total of 5 of 10 treatment-related deaths occurring as a result of haemoptysis, all in the bevacizumab group [100]. Indeed, the median survival was 12.3 months in the group assigned to chemotherapy plus bevacizumab, as compared with 10.3 months in the chemotherapy-alone group ( $p=0.003)$. In the former study, VEGF levels did not correlate with overall survival. In addition to distant invasion, another characteristic gained by metastatic cells is the adaptive and disorganised formation of new blood vessels with ultrastructural abnormalities from pre-existing vessels possibly mediated by VEGF. Conversely, a recent study found that the treatment with cisplatin/gemcitabine/bevacizumab (PGB) was superior to erlotinib-bevacizumab treatment in patients displaying a mesenchymal phenotype (low E-cadherin or high vimentin), but not in those with an epithelial phenotype (high E-cadherin or low vimentin) [101]. VEGF binds to precursors of endothelial cells via transmembrane receptors of the tyrosine kinase family, flt-1, and FLK-1/KDR, promoting the expansion, migration, and differentiation of vascular networks [23, 95].

In previous research on coculture in vitro experiments by injecting human A375 parental cells into the internal carotid artery of nude mice, astrocytes were found to be involved as critical protectors of the tumour cells from 5-fluorouracil and cisplatin-induced apoptosis in human melanoma cells [102]. Moreover, Chu et al. found that astrocytes produce SDF-1a, IL-3, IL-6, interferon-y, TNF-a, TGF- $\beta$, and PDGF, which the tumour cells use to promote survival, growth, and potentially organ-specific metastasis $[1,3]$. The essential role of the tumour microenvironment in cancer progression, the paracrine growth factors involved, and the interactions between tumour cells and the brain microenvironment have been elegantly demonstrated by isolation of a brain-specific metastatic variant of the MDA-MB-231 human breast cancer cell line, one of the most metastatic cell lines, through repeated selection in vivo by Bos et al. [38]. In an early study investigating the molecular mechanisms of angiogenesis in experimental brain metastasis, it was found that tumour cells with high rates of metastatic spread to the brain overexpressed significantly higher levels of VEGF activity than tumour cells with low rates of brain metastasis, and also suggesting that VEGF is necessary but not sufficient for the production of brain metastases [103].

\section{Therapeutic potential of TGF- $\beta 1$ inhibitors in lung adenocarcinoma}

Targeted therapies have a response rate of up to $80 \%$, but progression almost always occurs within one to two years. Traditional chemotherapy has a response rate of about 20\% with one-year survival rates near to $30 \%$. TGF- $\beta 1$ is the most abundant isoform in mammals. At present there is a prospective randomised trial exploring the therapeutic options. For example, there are several pharmacokinetic approaches and ongoing trials that interfere with the EMT mechanism and the migration of cancer cells induced by TGF- $\beta 1$, such as BIX02189.

The downregulation or inhibition of TGF- $\beta 1$ (the most abundant isoform in mammals) receptors results in cellular resistance to the usual suppressive effects of the TGF- $\beta 1$ ligand, contributing to cancer development and apoptosis. Existing therapies that target TGF- $\beta 1$ receptors in a variety of human cancers for potential clinical use are based on antisense molecules (oligonucleotides), monoclonal antibodies against the receptors, soluble TGF- $\beta$ inhibitors, receptor kinase inhibitors, peptide aptamers, and human vaccines. These therapies were designed to act against specific targets, including Smad proteins and other targets downstream the TGF- $\beta 1$ signalling pathway. Nonetheless, despite its initial promise, currently many targeted therapies either against ligand, ligand-receptor, or intracellular level of the TGF- $\beta 1$ on lung adenocarcinoma, which are considered relevant, have been discarded from both in vitro and in vivo research.

Due to brain metastasis from lung adenocarcinoma and its highly complex microenvironment, it is difficult to find a fully comprehensive and effective therapeutic approach. The ability of therapeutic strategies targeting the activating or inhibitory receptors on TGF- $\beta 1$ to stop or reverse the EMT has been reported in A549 lung cancer cells $[15,104]$. In an experimental model on cultured human A549 cells investigating the involvement of ERK1/2 in phosphorylation of Smad3 linker region and EMT induced by TGF- $\beta 1$, it was found that kaempferol, a common natural flavonoid, acts as a potent antitumour growth agent by reversing TGF- $\beta 1$-mediated Snail induction and E-cadherin repression by weakening Smad3 binding to Snail promoter [105].

The role of the immune system in cancer progression has been studied for decades. Programmed death-ligand 1 (PD-L1) is a 40kDa type transmembrane protein, a member of the B7-CD28 immunoglobulin superfamily expressed on activated T-cells and B-cells, with an important role in mediating immune evasion in the tumour microenvironment closely related to the EMT process through a negative feedback loop. An outstanding recent study reported that the AKT, ERK, and TAK1 pathways regulated the expression of PD-L1 by mediating transportation of the transcription factor Stat3 and the p65 subunit of NF-kB from the cytoplasm to the nucleus, with such findings determined by western blotting and flow cytometric analyses [63].

Recently, by investigating volatile anaesthetic agents such as sevoflurane on cell viability and chemoresistance to cisplatin on LA A549 cells in an in vitro study, it was found that sevoflurane positively upregulated expression 
of nuclear Smad3 and TGF- $\beta 1$ with enhanced chemosensitivity to cisplatin but without effect on migration of A549 cells [44].

As previously mentioned, MEK/ERK5 (mitogen-activated protein kinase/extracellular signal-regulated kinase [ERK]5) signalling pathway, strongly linked to chemoresistance, encoded by MAP2K5, can be inhibited in human A549 lung cancer cells. BIX02189, a pharmacological inhibitor of the MEK5 signalling pathway, has been shown to significantly interfere with the EMT mechanism and in the migration of cancer cells induced by TGF- $\beta 1[27,56$, 64]. Furthermore, a previous study has further shown that cyclooxygenase-2 (COX-2) inhibitors enhance the MEK/ Snail1 signalling and lead to metastasis and chemoresistance via EMT induction in NSCLC [106].

Antisense oligonucleotides are a class of molecule that can specifically bind to RNA target molecules in order to manipulate gene expression. Unfortunately, there is no therapy approved by the US Food and Drug Administration for the treatment of LAs, but it may be a feasible approach to minimise levels of TGF- $\beta 1$ [73].

Fresolimumab, a human neutralising antibody of all mammalian active isoforms of TGF- $\beta$, commonly well tolerated, was developed initially in a phase I study to treat patients with advanced melanoma and renal cell carcinoma, with contradictory results [107].

\section{Conclusions}

Additional challenges will be faced, shaping the design of clinical trials in cancer biology and pathway targets. In recent years, several researchers have focused on developing TGF- $\beta 1$ pathway inhibitors, and immense effort has been made to investigate methods for selective eradication of therapeutically resistant cells in lung adenocarcinoma.

The goal of the present review was to give a brief and non-conclusive overview of the most recent data about the implication of TGF- $\beta 1$ receptors and ligands on lung adenocarcinoma related to the contextual EMT and the multiple therapeutic strategies that have been investigated for improving the effectiveness of TGF- $\beta 1$ inhibitors. As noted, TGF- $\beta 1$ is frequently identified as being integral in regulating invasiveness and metastasis in a variety of human cancer types and represents an opportunity to develop therapeutic approaches.

The authors declare no conflict of interest.

\section{References}

1. Chu JE, Allan AL. The role of cancer stem cells in the organ tropism of breast cancer metastasis: a mechanistic balance between the "Seed" and the "Soil"? Int J Breast Cancer 2012; 2012: 209748.

2. Reka AK, Chen G, Jones RC, et al. Epithelial-mesenchymal transition-associated secretory phenotype predicts survival in lung cancer patients. Carcinogenesis 2014; 35: 1292-1300.

3. Sierra A, Price JE, García-Ramirez M, Méndez O, López L, Fabra A. Astrocyte-derived cytokines contribute to the metastatic brain specificity of breast cancer cells. Lab Invest 1997; 77: 357-368.

4. Testa U, Castelli G, Pelosi E. Lung Cancers: Molecular Characterization, Clonal Heterogeneity and Evolution, and Cancer Stem Cells. Cancers (Basel) 2018; 10: E248.
5. Tong B, Wang M. Circulating tumor cells in patients with lung cancer: developments and applications for precision medicine. Future Oncol 2019; 15: 2531-2542.

6. Tsubakihara Y, Moustakas A. Epithelial-mesenchymal transition and metastasis under the control of transforming growth factor $\beta$. Int J Mol Sci 2018; 19: 3672.

7. Ungefroren H, Witte D, Fiedler C, Gädeken T, Kaufmann R, Lehnert H, Gieseler F, Rauch B. The Role of PAR2 in TGF- $\beta 1$-Induced ERK activation and cell Motility. Int J Mol Sci 2017; 18: 2776.

8. Wu X, Ma W, Zhou Q, et al. AXL-GAS6 expression can predict for adverse prognosis in non-small cell lung cancer with brain metastases. J Cancer Res Clin Oncol 2017; 143: 1947-1957.

9. Lu X, Gao J, Zhang Y, Zhao T, Cai H, Zhang T. CTEN induces epithelial-mesenchymal transition (EMT) and metastasis in non-small cell lung cancer cells. PLoS One 2018; 13: 1-9.

10. Lu Y, Ma J, Li Y, et al. CDP138 silencing inhibits TGF- $\beta / S m a d$ signaling to impair radioresistance and metastasis via GDF15 in lung cancer. Cell Death Dis 2017; 8: e3036.

11. Schiller JH, Harrington D, Belani CP, Langer C, Sandler A, Krook J, Zhu J, Johnson DH; Eastern cooperative oncology group. Comparison of four chemotherapy regimens for advanced non-small-cell lung cancer. N Engl J Med 2002; 346: 92-98.

12. Travis WD, Brambilla E, Nicholson AG, et al. The 2015 world health organization classification of lung tumors: Impact of genetic, clinical and radiologic advances since the 2004 classification. J Thorac Oncol 2015; 10: 1243-1260.

13. Xu J, Wang AH, Oses-Prieto J, et al. Arginine methylation initiates BMP-induced smad signaling. Mol Cell 2013; 51: 5-19.

14. Yang X, Lin D. [Changes of 2015 WHO histological classification of lung cancer and the clinical significance]. Zhongguo Fei Ai Za Zhi 2016; 19: 332-336.

15. Jo E, Park SJ, Choi YS, Jeon WK, Kim BC. Kaempferol suppresses transforming growth factor- $\beta 1$-induced epithelial-to-mesenchymal transition and migration of A549 lung cancer cells by inhibiting Akt1-mediated phosphorylation of Smad3 at Threonine-179. Neoplasia 2015; 17: 525-537.

16. Kujawa A, Olias P, Bottcher A, Klopfleisch R. Thyroid transcription factor-1 is a specific marker of benign but not malignant feline lung tumours. J Comp Pathol 2014; 151: 19-24.

17. Maxfield KE, Taus PJ, Corcoran K, et al. Comprehensive functional characterization of cancer-testis antigens defines obligate participation in multiple hallmarks of cancer. Nat Commun 2015; 6: 1-15.

18. Faroogi AA, Tang JY, Li RN, et al. Epigenetic mechanisms in cancer: push and pull between kneaded erasers and fate writers. Int Nanomedicine 2015; 10: 3183.

19. Liu J, Huang B, Xiu Z, Zhou Z, Liu J, Li X, Tang X. PI3K/Akt/HIF-1 $\alpha$ signaling pathway mediates HPV-16 oncoprotein-induced expression of EMT-related transcription factors in non-small cell lung cancer cells. J Cancer 2018; 9: 3456-3466

20. Milano A, Mazzetta F, Valente S, et al. Molecular detection of EMT markers in circulating tumor cells from metastatic non-small cell lung cancer patients: Potential Role in Clinical Practice. Anal Cell Pathol (Amst) 2018; 2018: 3506874.

21. Piljić Burazer M, Mladinov S, Ćapkun V, Kuret S, Glavina Durdov M. The utility of thyroid transcription factor 1 (TTF-1), napsin A, excision repair cross-complementing 1 (ERCC1), anaplastic lymphoma kinase (ALK) and the epidermal growth factor receptor (EGFR) expression in small biopsy in prognosis of patients with lung adenocarcinoma - A retrograde single-center study from Croatia. Med Sci Monit 2017; 23: 489-497.

22. Risolino M, Mandia N, lavarone F, et al. Transcription factor PREP1 induces EMT and metastasis by controlling the TGF -SMAD3 pathway in non-small cell lung adenocarcinoma. Proc Natl Acad Sci 2014; 111: E3775-E3784.

23. Seystahl K, Tritschler I, Szabo E, Tabatabai G, Weller M. Differential regulation of TGF- $\beta$ 2-induced, ALK-5-mediated VEGF release by Smad2/3 versus SMAD1/5/8 signaling in glioblastoma. Neuro Oncol 2015; 17: 254-265.

24. Wang HB, Zhang HJ, Su JM, Zhou WY, Wang HY, Chen XF. [Epithelial-mesenchymal transition (EMT) and its effect on microRNA expression in lung cancer]. Zhonghua Zhong Liu Za Zhi 2011; 33: 590-593. 
25. Zhang X, Zhang P, Shao M, Zang X, Zhang J, Mao F, Qian H, Xu W. SALL4 activates TGF- $\beta$ /SMAD signaling pathway to induce EMT and promote gastric cancer metastasis. Cancer Manag Res 2018; 10: 4459-4470.

26. Nickel J, Dijke ten P, Mueller TD. TGF- $\beta$ family co-receptor function and signaling. Acta Biochim Biophys Sin (Shanghai) 2018; 50: 12-36.

27. Park SJ, Choi YS, Lee S, Lee YJ, Hong S, Han S, Kim BC. BIX02189 inhibits TGF- $\beta 1$-induced lung cancer cell metastasis by directly targeting TGF- $\beta$ type I receptor. Cancer Lett 2016; 381: 314-322.

28. Ren Y, Yin Z, Li K, Wan Y, Li X, Wu W, Guan P, Zhou B. TGF $\beta$-1 and TGFBR2 polymorphisms, cooking oil fume exposure and risk of lung adenocarcinoma in Chinese nonsmoking females: a case control study. BMC Med Genet 2015; 16: 22

29. Wang A, Lu C, Ning Z, et al. Tumor-associated macrophages promote Ezrin phosphorylation-mediated epithelial-mesenchymal transition in lung adenocarcinoma through FUT4/LeY up-regulation. Oncotarget 2017; 8: 28247-28259.

30. Neuzillet C, de Gramont A, Tijeras-Raballand A, de Mestier L, Cros J, Faivre $S$, Raymond E. Perspectives of TGF- $\beta$ inhibition in pancreatic and hepatocellular carcinomas. Oncotarget 2014; 5: 78-94.

31. Du S, Bouquet S, Lo CH, Pellicciotta I, Bolourchi S, Parry R, Barcellos-Hoff MH. Attenuation of the DNA damage response by trans forming growth factor-beta inhibitors enhances radiation sensitivity of non-small-cell lung cancer cells in vitro and in vivo. Int J Radiat Oncol Biol Phys 2015; 91: 91-99.

32. Abnaof K, Mallela N, Walenda G, et al. TGF- $\beta$ stimulation in human and murine cells reveals commonly affected biological processes and pathways at transcription level. BMC Syst Biol 2014; 8: 55.

33. Ahmadi A, Najafi M, Farhood B, Mortezaee K. Transforming growth factor $\beta$ signaling: Tumorigenesis and targeting for cancer therapy. J Cell Physiol 2019; 234: 12173-12187.

34. Balzac F, Avolio M, Degani S, Kaverina I, Torti M, Silengo L, Small JV, Retta SF. E-cadherin endocytosis regulates the activity of Rap1: a traffic light GTPase at the crossroads between cadherin and integrin function. J Cell Sci 2005; 118: 4765-4783.

35. Barciszewska AM. MicroRNAs as efficient biomarkers in high grade gliomas. Folia Neuropathol 2017; 4: 369-374.

36. Bellomo C, Caja L, Moustakas A. Transforming growth factor $\beta$ as regulator of cancer stemness and metastasis. Br J Cancer 2016; 115: 761-769.

37. Blandin AF, Renner G, Lehmann M, Lelong-Rebel I, Martin S, Dontenwill M. $\beta 1$ Integrins as Therapeutic Targets to Disrupt Hallmarks of Cancer. Front Pharmacol 2015; 6: 279.

38. Bos PD, Zhang XH, Nadal C, et al. Genes that mediate breast cancer metastasis to the brain. Nature 2009; 459: 1005-1009.

39. Calon A, Tauriello DV, Batlle E. TGF-beta in CAF-mediated tumor growth and metastasis. Semin Cancer Biol 2014; 25: 15-22.

40. Carbonell WS, Ansorga O, Sibson N, Muschel R. The vascular base ment membrane as "soil" in brain metastasis. Plos One 2009; 4: e5857.

41. Celià-Terrassa T, Bastian C, Liu D, et al. Hysteresis control of epithe lial-mesenchymal transition dynamics conveys a distinct program with enhanced metastatic ability. Nat Commun 2018; 9: 5005.

42. Chan R, Sethi P, Jyoti A, McGarry R, Upreti M. Investigating the radioresistant properties of lung cancer stem cells in the context of the tumor microenvironment. Radiat Res 2016; 185: 169-181.

43. Chen Z, Fillmore CM, Hammerman PS, Kim CF, Wong KK. Nonsmall-cell lung cancers: a heterogeneous set of diseases. Nature Publishing Group. Nat Rev Cancer 2014; 14: 535-546.

44. Ciechanowicz S, Zhao H, Chen Q, Cui J, Mi E, Mi E, Lian Q, Ma D. Differential effects of sevoflurane on the metastatic potential and chemosensitivity of non-small-cell lung adenocarcinoma and renal cell carcinoma in vitro. Br J Anaesth 2018; 120: 368-375.

45. Dolivo DM, Larson SA, Dominko T. Crosstalk between mitogen-activated protein kinase inhibitors and transforming growth fac tor-beta signaling results in variable activation of human dermal fibroblast. Int J Mol Med 2019; 43: 325-335.

46. Eichler AF, Chung E, Kodack DP, Loeffler JS, Fukumura D, Jain RK. The biology of brain metastases - translation to new therapies. Nat Rev Clin Oncol 2011; 8: 344-356.
47. Elmansuri AZ, Tanino MA, Mahabir R, et al. Novel signaling collaboration between TGF- $\beta$ and adaptor protein Crk facilitates EMT in human lung cancer. Oncotarget 2016; 7: 27094-27107.

48. Ganguly KK, Pal S, Moulik S, Chatterjee A. Integrins and metastasis. Cell Adh Migr 2013; 7: 251-261.

49. Gottlieb E, Vander Heiden MG, Thompson CB. BCl-xL prevents the initial decrease in mitochondrial membrane potential and subsequent reactive oxygen species production during tumor necrosis factor alpha-induced apoptosis. Mol Cell Biol 2000; 20: 5680-5689.

50. Gow CH, Liu YN, Li HY, et al. Oncogenic function of a KIF5B-MET fusion variant in non-small cell lung cancer. Neoplasia 2018; 20: 838-847.

51. Haga RB, Ridley AJ. Rho GTPases: Regulation and roles in cancer cell biology. Small GTPases 2016; 7: 207-221.

52. Halvorsen AR, Bjaanæs M, LeBlanc M, et al. A unique set of 6 circulating microRNAs for early detection of non-small cell lung cancer. Oncotarget 2016; 7: 37250-37259.

53. Han G, Li F, Singh TP, Wolf P, Wang X-J. The pro-inflammatory role of TGF: a paradox? Int J Biol Sci 2012; 8: 228-235.

54. Hanssen A, Riebensahm C, Mohme M, et al. Frequency of circulating tumor cells (CTC) in patients with brain metastases: Implications as a risk assessment marker in oligo-metastatic disease. Cancers (Basel) 2018; 10: pii: E527.

55. Hoang VT, Yan TJ, Cavanaugh JE, Flaherty PT, Beckman BS, Burow ME. Oncogenic signaling of MEK5-ERK5. Cancer Lett 2017; 392: 51-59.

56. Isogaya K, Koinuma D, Tsutsumi S, Saito R-A, Miyazawa K, Aburatani H, Miyazono K. A Smad3 and TTF-1/NKX2-1 complex regulates Smad4-independent gene expression. Cell Res 2014; 24: 9941008

57. Ju L, Zhou C. Association of integrin betal and c-MET in mediating EGFR TKI gefitinib resistance in non-small cell lung cancer. Cancer Cell Int 2013; 13: 15.

58. Kalluri R, Weinberg RA. The basics of epithelial-mesenchymal transition. J Clin Invest 2009; 119: 1420-1428.

59. Katz LH, Li Y, Chen JS, Muñoz NM, Majumdar A, Chen J, Mishra L. Targeting TGF- $\beta$ signaling in cancer. Expert Opin Ther Targets 2013; 17: 743-760.

60. Kim J, Hwan Kim S. CK2 Inhibitor CX-4945 blocks TGF- $\beta 1$-induced epithelial-to-mesenchymal transition in A549 human lung adenocarcinoma Cells. Plos One 2013; 8: e74342.

61. Kim J, Moon S-H, Kim BT, Chae CH, Lee JY, Kim SH. A novel aminothiazole KY-05009 with potential to inhibit Traf2- and Nck-interacting kinase (TNIK) attenuates TGF- $\beta 1$-mediated epithelial-to-mesenchymal transition in human lung adenocarcinoma A549 Cells. PLoS One 2014; 9: e110180.

62. Kim YE, Kim JO, Park KS, Won M, Kim KE, Kim KK. Transforming growth factor- $\beta$-induced RBFOX3 inhibition promotes epithelial-mesenchymal transition of lung cancer cells. Mol Cells 2016; 39: 625-630.

63. Li F, Zhu T, Yue Y, Zhu X, Wang J, Liang L. Preliminary mechanisms of regulating PD-L1 expression in non-small cell lung cancer during the EMT process. Oncol Rep 2018; 40: 775-782.

64. Li J, Ao J, Li K, et al. ZNF32 contributes to the induction of multidrug resistance by regulating TGF- $\beta$ receptor 2 signaling in lung adenocarcinoma. Cell Death Dis 2016; 7: e2428.

65. Li L, Zhao GD, Shi Z, Qi LL, Zhou LY, Fu ZX. The Ras/Raf/MEK/ERK signaling pathway and its role in the occurrence and development of HCC. Oncol Lett 2016; 12: 3045-3050.

66. Mizutani A1, Koinuma D, Tsutsumi S, et al. Cell type-specific target selection by combinatorial binding of $5 \mathrm{mad} 2 / 3$ proteins and hepatocyte nuclear factor 4 in hepG2 cells. J Biol Chem 2011; 286: 29848-29860.

67. Morikawa M, Koinuma D, Miyazono K, Heldin C-H. Genome-wide mechanisms of Smad binding. Oncogene 2012; 32: 1609-1615.

68. Zhang F, Ren CC, Liu L, Chen YN, Yang L, Zhang XA. HOXC6 gene silencing inhibits epithelial-mesenchymal transition and cell viability through the TGF- $\beta$ /smad signaling pathway in cervical carcinoma cells. Cancer Cell Int 2018; 18: 1-16.

69. Zhang H, Yang W, Yan J, et al. Loss of profilin 2 contributes to enhanced epithelial-mesenchymal transition and metastasis of colorectal cancer. Int J Oncol 2018; 53: 1118-1128. 
70. Zhang YX, Yan YF, Liu YM, et al. Smad3-related miRNAs regulated oncogenic TRIB2 promoter activity to effectively suppress lung ad enocarcinoma growth. Cell Death Dis 2016; 7: e2528.

71. Zhao J, Liu J, Lee J-F, et al. TGF- $\beta / S m a d 3$ pathway stimulates sphingosine-1 phosphate receptor 3 expression. J Biol Chem 2016; 291 : 27343-27353.

72. Zhao M, Mishra L, Deng CX. The role of TGF- $\beta / S M A D 4$ signaling in cancer. Int J Biol Sci 2018; 14: 111-123.

73. Le BT, Raguraman P, Kosbar TR, Fletcher S, Wilton SD, Veedu RN. Antisense oligonucleotides targeting angiogenic factors as potential cancer therapeutics. Mol Ther Nucleic Acids 2019; 14: 142-157.

74. Wang H, Zhu Y, Zhao M, et al. miRNA-29c suppresses lung cance cell adhesion to extracellular matrix and metastasis by targeting integrin $\beta 1$ and matrix metalloproteinase2 (MMP2). PLoS One 2013; 8: e70192.

75. Korkut A, Zaidi S, Kanchi RS, et al. A pan-cancer analysis reveals high-frequency genetic alterations in mediators of signaling by the TGF- $\beta$ superfamily. Cell Syst 2018; 7: 422-437.

76. Zhang C, Hao Y, Wang Y, Xu J, Teng Y, Yang X. TGF-B/SMAD4-regu lated InCRNA-LINP1 inhibits epithelial-mesenchymal transition in lung cancer. Int J Biol Sci 2018; 14: 1715-1723.

77. Li X, Wu X. MiR-21-5p promotes the progression of non-small-cell lung cancer by regulating the expression of SMAD7. Onco Targets Ther 2018; 11: 8445-8454.

78. Wang M, Jia M, Yuan K. MicroRNA-663b promotes cell prolifera tion and epithelial mesenchymal transition by directly targeting Smad7 in nasopharyngeal carcinoma. Exp Ther Med 2018; 16 3129-3134.

79. Sato T, Muramatsu T, Tanabe M, Inazawa J. Identification and characterization of transforming growth factor beta-induced in circu lating tumor cell subline from pancreatic cancer cell line. Cancer Sci 2018; 109: 3623-3633.

80. Shah S, Fourgeaud C, Derieux S, et al. The close relationship between heparanase and epithelial mesenchymal transition in gas tric signet-ring cell adenocarcinoma. Oncotarget 2018; 9: 33778 33787.

81. Shi J, Feng J, Xie J, et al. Targeted blockade of TGF- $\beta$ and IL-6/JAK2/ STAT3 pathways inhibits lung cancer growth promoted by bone marrow-derived myofibroblasts. Sci Rep 2017; 7: 8660.

82. Sun S, Schiller JH, Spinola M, Minna JD. New molecularly targeted therapies for lung cancer. J Clin Invest 2007; 117: 2740-2750.

83. Tang YN, Ding WQ, Guo XJ, Yuan XW, Wang DM, Song J. Epigenetic regulation of Smad2 and Smad3 by profilin-2 promotes lung cancer growth and metastasis. Nat Commun 2015; 6: 8230.

84. Tiwary S, Morales JE, Kwiatkowski SC, Lang FF, Rao G, McCarty JH. Metastatic Brain Tumors Disrupt the Blood-Brain Barrier and Alter Lipid Metabolism by Inhibiting Expression of the Endothelial Cell Fatty Acid Transporter Mfsd2a. Sci Rep 2018; 8: 8267

85. Abd El-Fattah AA, Sadik NAH, Shaker OG, Mohamed Kamal A. Single nucleotide polymorphism in Smad7 and CHi3L1 and colorectal cancer risk. Mediators Inflamm 2018; 2018: 9853192.

86. Pangas SA, Li X, Umans L, et al. Conditional deletion of Smad1 and Smad5 in somatic cells of male and female gonads leads to metastatic tumor development in mice. Mol Cell Biol 2008; 28: 248-257.

87. Toofan P, Busch C, Morrison H, O'Brien S, Jørgensen H, Copland M, Wheadon $\mathrm{H}$. Chronic myeloid leukaemia cells require the bone morphogenic protein pathway for cell cycle progression and self-renewal. Cell Death Dis 2018; 9: 927.

88. Matveeva A, Kovalevska L, Kholodnyuk I, Ivanivskaya T, Kashuba E. The TGF-beta-Smad pathway is inactivated in cronic lymphocytic leukemia cells. Exp Oncol 2017; 39: 286-290.

89. Othman N, In LLA, Harikrishna JA, Hasima N. Bcl-xL Silencing induces alterations in hsa-miR-608 expression and subsequent cell death in A549 and SK-LU1 human lung adenocarcinoma cells. PLoS One 2013; 8: e81735.

90. Niederst MJ, Engelman JA. Bypass mechanisms of resistance to receptor tyrosine kinase inhibition in lung cancer. Sci Signal 2013 6: re6.

91. Yu JR, Tai Y, Jin Y, Hammell MC, Wilkinson JE, Roe JS, Vakoc CR, Van Aelst L. TGF- $\beta /$ Smad signaling through DOCK4 facilitates lung adenocarcinoma metastasis. Genes Dev 2015; 29: 250-261.

92. Pekmezci M, Perry A. Neuropathology of brain metastases. Surg Neurol Int 2013: 4: S245-255.
93. Ko JH, Nam D, Um JY, Jung SH, Sethi G, Ahn KS. Bergamottin suppresses metastasis of lung cancer cells through abrogation of diverse oncogenic signaling cascades and epithelial-to-mesenchymal transition. Molecules 2018; 23: pii: E1601.

94. Li M, Marin-Muller C, Bharadwaj U, Chow KH., Yao Q, Chen C. MicroRNAs: control and loss of control in human physiology and disease. World J Surg 2009; 33: 667-684.

95. Liu L, Bi N, Wu L, et al. MicroRNA-29c functions as a tumor suppressor by targeting VEGFA in lung adenocarcinoma. Mol Cancer 2017; 16: 50.

96. Kölbl AC, Jeschke U, Andergassen U. The significance of epithelial-to-mesenchymal transition for circulating tumor cells. Int J Mo Sci 2016; 17: 1-12.

97. Lamouille S, Xu J, Derynck R. Molecular mechanisms of epithelialmesenchymal transition. Nat Rev Mol Cell Biol 2014; 15: 178-196.

98. Ramakrishna R, Rostomily R. Seed, soil, and beyond: the basic biology of brain metastasis. Surg Neurol Int 2013; 4 (Suppl 4): S256-264

99. Wrobel JK, Toborek M. Blood-brain barrier remodeling during brain metastasis formation. Mol Med 2016; 22: 32-40.

100.Sandler A, Gray R, Perry MC, Brahmer J, Schiller JH, Dowlati A Lilenbaum R, Johnson DH. Paclitaxel-carboplatin alone or with bevacizumab for non-small-cell lung cancer. N Engl J Med 2006; 355: 2542-2550.

101. Villalobos M, Czapiewski P, Reinmuth N, et al. Impact of EMT in stage IIIB/IV NSCLC treated with erlotinib and bevacizumab when compared with cisplatin, gemcitabine and bevacizumab. Oncol Lett 2019; 17: 4891-4900.

102. Lin Q, Balasubramanian K, Fan D, et al. Reactive astrocytes protect melanoma cells from chemotherapy by sequestering intracellular calcium through gap junction communication channels. Neoplasia 2015; 12: 748-754.

103. Yano S, Shinohara H, Herbst RS, et al. Expression of vascular endothelial growth factor is necessary but not sufficient for production and growth of brain metastasis. Cancer Res 2000; 60: 4959-4967.

104.Pan P, Tan H, Wang X, Liu B, Yang X, Li H. Oncogenic role of epithelial cell transforming sequence 2 in lung adenocarcinoma cells. Exp Ther Med 2016; 12: 2088-2094.

105. Samanta D, Gonzalez AL, Nagathihalli N, Ye F, Carbone DP, Datta PK. Smoking attenuates transforming growth factor-mediated tumor suppression function through downregulation of Smad3 in lung cancer. Cancer Prev Res 2012; 5: 453-463.

106. Wang Z, Fan Z, Jiang H, Ou J. Selective Cox-2 inhibitor celecoxib induces epithelial-mesenchymal transition in human lung cancer cells via activating MEK-ERK signaling. Carcinogenesis 2013; 34 638-646.

107. Oude Munnink TH, Arjaans ME, Timmer-Bosscha H, et al. PET with the $89 \mathrm{Zr}$-labeled transforming growth factor- $\beta$ antibody fresolimumab in tumor models. J Nucl Med 2011; 52: 2001-2008.

\section{Address for correspondence}

\section{Kelvin Piña Batista}

Department of Neurosurgery

Central University Hospital of Asturias

Avenida de Roma 33011

Oviedo, Spain

e-mail: pineappledr@gmail.com

Submitted: 7.06.2019

Accepted: 4.11.2019 